\title{
Neuromuscular diseases and Covid-19: Advices from scientific societies and early observations in Italy
}

\author{
Corrado Angelini (1), Gabriele Siciliano (2) \\ (1) IRCCS San Camillo Hospital, Venice, Italy; (2) Neurological Clinics, University Hospital of \\ Pisa, Italy \\ This article is distributed under the terms of the Creative Commons Attribution Noncommercial License (CC BY-NC 4.0) which permits \\ any noncommercial use, distribution, and reproduction in any medium, provided the original author(s) and source are credited.
}

\begin{abstract}
The risk of a severe course of COVID-19 is increased in patients suffering with Neuromuscular disorders (NMD) due to the following comorbidities: muscular weakness of the chest and diaphragm, use of ventilator supports and/or presence of tracheostoma, weak airway clearance, cardiac involvement, rhabdomyolysis, comoribities, steroid and immunosuppressant treatments. NMD display varying levels of disability in people with the same diagnosis, thus it is difficult to give COVID-19 related general recommendations. Present advicess were designed for patients, caregivers, general neurologists and non-specialist medical providers. They address frequently asked questions and basic service requirements and are supported by a series of in-depth references. In this truly unprecedented situation, the clinical management of neuromuscular patients during the COVID-19 epidemics - taking into account the related difficulties (patients who have suspended ERT, difficulty in contacting the doctors, etc.) - we propose to use a telemedicine device, i.e. the AIGkit application (AIGkit app), promoted and developed in 2018 by Fabrizio Seidita on behalf of the Italian Glycogenosis Association (AIG). The app was born to allow patients with Pompe disease to receive as far as possible continuous monitoring of their health. The support of all colleagues of the Italian Association for Myology (AIM) should extend its use to all NMD patients and beyond.
\end{abstract}

Key Words: Covid-19, neuromuscular disorders, advice from scientific societies, AIGkit application, AIM.

Eur J Transl Myol 30 (2): 286-290, 2020

The category of neuromuscular disorders (NMD) covers a wide range of different diagnoses with widely varying levels of disability even in people with the same diagnosis. Though it is difficult to make general, nothing to say specific, recommendations, those that will be described might be applied to numerous neuromuscular diseases. They were designed by a team of experts of the Muscle World Society primarily for patients, caregivers, general neurologists and non-specialist medical providers, to inform neuromuscular specialists on frequently asked questions and basic service requirements. Anyhow, in-depth reference links are provided.

\section{Are people with neuromuscular disease (NMD) at higher risk?}

National neurological associations and neuromuscular networks (Association of British Neurologists, European Reference Network EURO-NMD, others) have produced guidance on the impact of Covid-19 $19^{1}$ on NMD and their management. The documents define the risk of a severe course of Covid-19 as high or moderately high in all NMD except the mildest forms. Features conferring a high risk of severe disease include, for example:

- Muscular weakness of the chest or diaphragm, resulting in respiratory volumes less than 60\% predicted $(\mathrm{FVC}<60 \%)$, especially in patients with kyphoscoliosis

- Use of ventilation via mask or tracheotomy

- Weak cough and weak airway clearance due to oropharyngeal weakness

- Presence of tracheostoma

- Cardiac involvement (and/or on medication for heart involvement)

- Risk of deterioration with fever, fasting or infection

- Risk of rhabdomyolysis with fever, fasting or infection

- Concomitant diabetes and obesity 


\section{Covid-19 epidemic and neuromuscular disorders}

Eur J Transl Myol 30 (2): 286-290, 2020

- Patients taking steroids and undergoing immunosuppressant treatment

Documents supporting advices in Sections 1 to 9 are available at:

https://www.worldmusclesociety.org ${ }^{2}$

https://ern-euro-nmd.eu/covid-19-and-people-withneuromuscular-disorders-world-muscle-societyposition-and-advice/ ${ }^{3}$

https://ern-euro-nmd.eu/ ${ }^{4}$

https://www.ema.europa.eu/ ${ }^{5}$

https://www.eanpages.org/2020/04/08/ean-

scientific-panel-muscle-nmj-disorders $/{ }^{6}$

https://www.theabn.org/page/COVID-19 ${ }^{7}$

https://www.enmc.org ${ }^{8}$

https://neuromuscularnetwork.ca/news/covid-19and-neuromuscular-patients-la-covid-19-et-lespatients-neuromusculaires $/{ }^{9}$

https://www.youtube.com/watch?v=3DKEeRV8alA $\&$ feature=youtu.be ${ }^{10}$

http://www.eamda.eu/2020/03/19/coronaviruscovid-19-information-for-people-with-nmd/ ${ }^{11}$

https://www.gov.uk/government/publications/guidan ce-on-shielding-and-protecting-extremelyvulnerable-persons-from-covid-19/ ${ }^{12}$

2. What do people with NMD need to do to avoid infection?

Covid-19 spreads infection through droplets when an infected person coughs, sneezes or talks, or potentially via touching a surface carrying infectious droplets. People with NMD and a high risk of a severe course of Covid-19 infection, as defined above, should undertake the following precautions:

- Social distancing of at least 2 meters (6 feet) is a minimum requirement. For high risk individuals (as defined in 1.), self-isolation is advised. Official advice on how to self-isolate should be followed.

- People are encouraged to work from home or stagger their working times if possible.

- Avoid large gatherings and public transport. People in general are urged to limit visits to vulnerable persons.

- Frequent hand-washing (20 seconds with soap and warm water), use of $60 \%$ alcohol-based hand sanitizers, and surface disinfection are crucial.

- Caregivers should be in-house, if possible. Essential visiting care givers (for instance, providers of backup support for ventilatory assistance) should wear face masks and adequate PPE according to up to date official guidance, to prevent passing on the virus.

- Visiting physiotherapy is discouraged, however, physiotherapists should provide advice on maintaining physical activity remotely, via phone or videolink.

- It is important to be prepared for all eventualities including when assistants are absent due to illness or quarantine. The person responsible for organizing home care should have an overview of the personnel situation at all times. Plans should be made for how to best meet the needs of the individual without resorting to hospitalization.

3. What consequences does the risk of Covid-19 infection have for treatments used in people with NMD?

- Patients must ensure they have an adequate supply of medication and of ventilatory support equipment for a period of prolonged isolation (at least 1 month supply).

- Patients and caregivers should make use of online and telephone -based for pharmacy and equipment ordering and delivery services.

- Patients and carers need to be comfortable with emergency procedures specific to their condition and their equipment.

- DMD patients on steroid regimens should continue their medication. Steroids must never be stopped suddenly, and there may be a need to increase the steroid dose when necessary.

- Immunosuppression in inflammatory muscle disease, myasthenia gravis, and peripheral nerve disease should not be discontinued except under specific circumstances and after consultation with the neuromuscular specialist.

- Isolation requirements may impact on treatment regimens requiring hospital procedures (i.e. nursinersen-Spinraza), alglucosidase alfa (Myozyme), intravenous immunoglobulin (IVIg) and rituximab infusions or treatments related to clinical trials). These treatments should not be stopped, but where possible moved to a non-hospital setting (home-visiting or outreach nurses), for which cooperation with manufacturing companies may be negotiated. IVIg can be changed to subcutaneous immunoglobulin.

4. What needs to be done to assure ventilatory services during self-isolation (LVR bags, home ventilators etc.)

- Backup and advice hotlines should be offered by the patients' Neuromuscular Centres.

- Patients should have an alert card/medical bracelet providing the Neuromuscular Centre contact.

- Neuromuscular Centres should actively contact patients on ventilatory support to ensure they have relevant information and adequate equipment.

5. When should people with NMD seek admission if they develop symptoms of infection?

Inpatient admission should be avoided if possible, but should not be delayed when necessary. This can be a difficult decision. People with NMD need to be aware that: 
- Emergency services may be under severe pressure.

- Individual countries may have triaging procedures in place. These may affect the potential for intensive care admission for people with NMD who require ventilation. Specifically, the terms "incurable" and "untreatable" may be confused by medical staff. Neuromuscular disorders may be incurable, but they are not untreatable, and the implications for treatment decisions are very different.

- Use of patients' home equipment (i.e. ventilators) may be prohibited by some hospital infection-control policies, or require modifications. Ideally, there should be a back-up plan.

6. Can treatments for Covid-19 have effects on neuromuscular disease?

- Numerous specific treatments for Covid-19 are under investigation. Some of these can affect neuromuscular function significantly: for example, chloroquine and azithromycin are unsafe in myasthenia gravis, except when ventilatory support is available.

- Other treatments may have effects on specific neuromuscular diseases (in particular, metabolic, mitochondrial, myotonic and neuromuscular junction disorders), and anatomical peculiarities may influence options for treatment (e.g. prolonged prone ventilation)

- Experimental treatments for Covid-19 may be offered "compassionately", i.e. outside trial conditions. They should only be taken after consultation with the patient's neuromuscular specialist.

7. What should neuromuscular specialists do to assist Emergency Medical and Intensive Care decisions on admission to units, escalation of treatment, and ceilings of care in neuromuscular patients?

Decisions on patient admission to Intensive Care may be affected by anticipated or existing capacity problems. Triaging may have been instituted. This can have practical and ethical consequences.

- There must be close collaboration between neuromuscular and respiratory physicians.

- The neuromuscular specialist must be available to play a role in ensuring fair provision of intensive care to NMD patients.

- Ideally, neuromuscular specialists will have involved themselves in formulating hospital policies, decisionmaking algorithms and documentation forms.

- Neuromuscular specialists must develop guidelines for treatment that ensure patients remain at home as long as possible.

8. What patient support should neuromuscular centres provide?

Neuromuscular centres and specialist services should aim to provide the following:

- Patient hotlines staffed by neuromuscular care advisors, physiotherapists and other specialist personnel, with specialist physician backup (paediatric and adult).

- The possibility to continue routine clinics by structured telemedical phone and video links (for this, national and institutional data security regulations such as HIPAA approval may need modification).

- Outreach ventilatory support strategies should be provided.

- Strategies to maintain hospital-based treatments with minimal disruption.

- Neuromuscular specialists should be in discussion with their hospitals' Emergency, Medical and Intensive Care departments on restrictions for use of home NIV equipment.

- Neuromuscular specialists should support their hospital to define approved devices and ensure their availability (i.e. ICU mask systems with viral particle filters to permit use of patients' NIV machines in hospital).

- Liaison and shared care with Intensive Care services.

\section{Early Observations of Italian NMD Patients}

A female patient of 54 years, who lives nearby Milan, is suffering since age 36 from a spastic tetraparesis due to a mutation of AP5Z1 gene (two heterozygous variants p.Leu1153His and pArg 523 His) determining SPG48. She had a biopsy with mitochondrial abnormalities and type 2 myofiber atrophy, has difficulty walking since age 40 , and suffers from respiratory crises for which she is confined at home and ventilated by local health care personnel. This early March 2020, after the visit from her family doctor (Covid-19 affected from March 16, 2020), had loss of sense for smell, ageusia and respiratory crises from 1 to 8 of April. Now, April 14 2020, she is slowly recovering at home being still ventilated. Her family doctor, which was found Covid-19 positive, died on March 24 at age 69.

Two dysferlinopathy patients (a man aged 44 years, and a women 40 year old with proximo-distal weakness) have so far no change in health and motor ability, but deep anxiety reaction to Corona virus pandemia. In patients affected by LGMD 1F/D2 affected by Transportin-3 defect, that gives immunity to HIV, so far no Covid-19 infection is reported.

A Duchenne muscular dystrophy patient, with a deletion in dystrophin gene of exons 46-47, who started walking at 16 months, was treated since age 5 with Deflazacort and now is on prednisone and Dilatrend for his cardiomyopathy. He is now 39 years old and home ventilated. Although he has no symptoms, he is very worried.

His 40 year old cousin, a femal case affected by betasarcoglycanopathy is also continuing nocturnal home ventilation and follows home treatment assisted by her parents, in the area of Bozen, Italy, at $900 \mathrm{~m}$ altitude although she has borderline low FVC and is wheel-chairbound. 
A male patient, 41 yr-old, affected by myotonic dystrophy type 1, CTG expansion range E2 at the 3' $D M P K$ gene untranslated region at q13.3 locus on chromosome 19 , is presenting with a score 3 at MIRS scale. He presented hand myotonia when he was 35 and now is requiring not invasive ventilation, eight hours for day. During our surveillance campaign by remote consultancy ,due to Corona virus pandemia, when contacted by phone he said that he was not taking anymore ventilation because he had left his ventilator equipment at his girlfriend house, $20 \mathrm{~km}$ far away, and it was not possible for him to collect it,due to the general lockdown established from the authorities. He was not experiencing any anxiety, but we adviced him to inform the carabinieres about the need to collect the ventilator as soon as possible and in the meantime to keep in touch with the specialists by teleconsultation.

All these preliminary observations in a few Italian NMD patients both from neurogenic or myogenic origin and in NIV confirm that NIV should be done at home or in Subintesive Care Units, as soon as the patients are suspected of Covid-19 infection on clinical grounds, if they present fever, symptoms of anosmia or ageusia. Furthermore, for muscle dystrophy patients steroids and cardiac supportive drugs should be continued.

AIG-KIT - an application for smartphone,
promoted by the Italian Glycogenosis Association (AIG) and the Italian Association of Myology (AIM)

In this situation, never present before, the clinical management of neuromuscular patients due to the epidemic of COVID19 and the related difficulties (reports on patients who have suspended ERT, difficulty in contact with doctors, etc.), it seems to be rational to restore use of the AIGkit application (AIGkit app). AIGkit app was promoted and developed during 2018 by Fabrizio Seidita with the support of the Italian Glycogenosis Association (AIG, Associazione Italiana Glicogenosi). ${ }^{13}$ The app was designed to allow patients with Pompe disease to receive costant remote monitoring of their health by the medical staff. ${ }^{14-16}$ Briefly, AIGkit is an application (app) for smartphone, promoted by AIG in collaboration with AIM and created with the technical support of Vidiemme engineers. The aim is to allow a constant electronic interaction between patients and doctors, making patient management easier. This application, free downloaded on iOS, Android and Windows operating systems, is easy and intuitive to use, consisting in 4 interactive areas that the patient can access. The first area, called "To know my disease", contains information relating to Pompe disease (what is the disease, its epidemiology, how it is transmitted, clinical manifestations, the diagnostic process and the therapies available to date); the second area, called "News", currently not active, contains updates related to the disease; in the third area, "My clinical data", the patient can record his health status, the therapies and his psycho-emotional state by using dedicated questionnaires. In the fourth area, "Training program", the patient can find personalized "training sessions", which of course must be in agreement with his specialist's prescriptions. The home page also contains the contacts of the two associations taking part in the project, AIM and AIG, and an emergency area in which by a single button the patients can call the last telephone number or send short localization messages that automatically show their geographical position on Google map. In 2018, in a pilot phase, a first data collection by the app was successfully carried out by a selected voluntary group of patients. Given the current emergency from COVID-19, this telemedicine tool will be useful in monitoring at distance patients with muscle diseases in their state of health, in particular regarding the functional motor and respiratory parameters (found in "My clinical data" section) that can be thus sent to the referring physician. We kind request to colleagues to be proactive in spreading and sharing this initiative with the patients. The project will be soon present on the AIM website (www.miologia.org) under the item "AIG-kit app for patients with Pompe disease". We remain available for further information, technical details and any need at the e-mail address below. Thanking for the collaboration, a warm greeting from Prof. Gabriele Siciliano, Dott.ssa Giulia Ricci, Dott.ssa Costanza Simoncini, Dott.ssa Erika Schirinzi. Centro Diagnosi e Cura delle Malattie Muscolari - Riferimento e Coordinatore Nazionale Rete Europea Malattie Rare “ERN Euro-NMD”. Clinica Neurologica dell'Università di Pisa e Azienda Ospedaliera Universitaria Pisana, Ospedale S. Chiara, via Roma 67. I-56126 Pisa, Italy

E-mail: amb.neuromuscpisa@ao-pisa.toscana.it

\section{List of acronyms}

AIG - Italian Glycogenosis Association

AIGkit app - AIGkit application

AIM - Italian Association of Myology

AP5Z1 - Adaptor related protein complex 5, subunit

Z1. gene

CoV-2 - Coronavirus - 2

COVID-19 - the disease due to SARS-CoV-2 virus

CTG repeats - Cytidine, Thymine, Guanine repeats

DMD - Duchenne Muscular Dystrophy

$D M P K$ - Dystrophia Myotonica Protein Kinase

EURO-NMD - European Reference Network for NMD

ERT - Enzyme Replacement Treatment

FVC - Forced Vital Capacity

HIPPAA - Health Insurance Portability Accuntability Act

HIV - human immunodeficiency viruses

ICU - Intensive Care Unit

iOS - iPhone Operative System

IVIg - intravenous immunoglobulin

LGMD 1F/D2 - Limb Girdle Muscular Dystrophy type

$1 \mathrm{~F}$ or D2

LVR - Lung Volume Recruitment

MIRS scale - Muscular Impairment Rating Scale

NIV - Nocturnal Invasive Ventilation 


\section{Covid-19 epidemic and neuromuscular disorders}

Eur J Transl Myol 30 (2): 286-290, 2020

NMD - neuromuscular disorders

PPE - Personal Protective Equipment

SARS - Severe Acute Respiratory Syndrome

SPG48 - Spastic Paraplegia type 48

\section{Authors' contributions}

CA and GS drafted, finalized, and approved the paper.

\section{Acknowledgments}

Authors thank Colleagues of IRCCS - San Camillo Hospital, Venezia-Lido (Valentina Pegoraro e Roberta Marozzo) Italy, of AIG (Fabrizio Seidita), of AIM ( Giovanni Antonini and Massimiliano Filosto), and of the University Hospital of Pisa (Giulia Ricci, Costanza Simoncini and Erika Schirinzi), for sharing informations on COVID-19. Chiara Proietti and Francesca Carlini of Vidiemme, Milan, Italy provided technical support during development of the AIGkit application.

\section{Funding}

AFM grant 22392 (France) and Associazione Conquistando Escalones support CA. A\&C M-C Foundation for Translational Myology, Padova, Italy supports UC. "ERN Euro-NMD" and AIG-Associazione Italiana Glicogenosi support GS.

\section{Conflict of Interest}

The authors declare they have no conflicts of interest.

\section{Ethical Publication Statement}

We confirm that we have read the Journal's position on issues involved in ethical publication and affirm that this report is consistent with those guidelines.

\section{Corresponding Author}

Corrado Angelini, IRCCS San Camillo Hospital, Via Alberoni, 70 - 30126 Venice-Lido, Italy.

ORCID iD: 0000-0002-9554-8794

E-mail: corrado.angelini@unipd.it

\section{E-mail of co-author}

Gabriele Siciliano: gabriele.siciliano@unipi.it ORCID iD: : 0000-0002-6142-2384

\section{References}

1. Carraro U. 2020PMD, 30-years of Translational Mobility Medicine at the time of COVID-19 outbreak: Last-minute forewords from the editor. Eur J Transl Myol 2020;30:8966. Doi 10.4081/ejtm. 2019.8966

2. https://www.worldmusclesociety.org

3. https://ern-euro-nmd.eu/covid-19-and-people-withneuromuscular-disorders-world-muscle-societyposition-and-advice/

4. https://ern-euro-nmd.eu/

5. https://www.ema.europa.eu/

6. https://www.eanpages.org/2020/04/08/eanscientific-panel-muscle-nmj-disorders/

7. https://www.theabn.org/page/COVID-19

8. https://www.enmc.org

9. https://neuromuscularnetwork.ca/news/covid-19and-neuromuscular-patients-la-covid-19-et-lespatients-neuromusculaires/

10. https://www.youtube.com/watch?v=3DKEeRV 8 alA \& feature $=$ youtu.be

11. http://www.eamda.eu/2020/03/19/coronaviruscovid-19-information-for-people-with-nmd/

12. https://www.gov.uk/government/publications/ guidance-on-shielding-and-protecting-extremelyvulnerable-persons-from-covid-19/

13. Ricci G, Baldanzi S, Seidita F, et al. Italian GSD II group. A mobile app for patients with Pompe disease and its possible clinical applications. Neuromuscul Disord 2018;28:471-5. Doi: 10.1016/ j.nmd.2018.03.005. Epub 2018 Mar 12.

14. Apple - https://itunes.apple.com/it/app/aigkit/id11 56925493? $\mathrm{mt}=8$

15. Android - https://play.google.com/store/apps/deta ils?id=it.vidiemme.dist.aig.aigkit

16. Windows - https://www.microsoft.com/it-it/store/ p/aigkit/9nblggh554lp

Submitted: April 15, 2020

Revision submitted May 07, 2020 Accepted for publication: May 10, 2020 